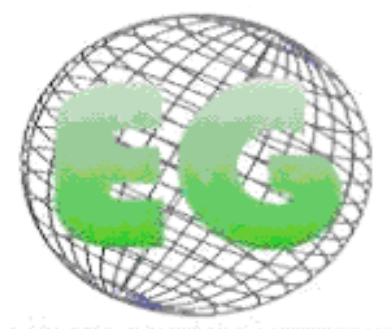

ISSN 1695.6141 $N^{\circ} 26$

\title{
Indicadores de calidad y seguridad del paciente en la enfermería de urgencias: un valor seguro.
}

Indicators of quality patient safety in emergency nursing: a safe value

*Ortells Abuye, N., "Paguina Marcos, M.

\author{
*Enfermeras. Servicio de Urgencias del Hospital de Palamós. Serveis de Salut Integrats Baix Empordà \\ (SSIBE). Girona. E-mail: nortells@ssibe.cat \\ Palabras clave: calidad de la atención de salud; seguridad; atención de enfermería; urgencias médicas. \\ Keywords: quality of health care; safety; nursing care; emergencies.
}

\section{RESUMEN}

Introducción: La calidad de los servicios de enfermería se podría definir como la atención oportuna, personalizada, humanizada, continua y eficiente que realiza el personal de enfermería, de acuerdo con los estándares definidos para una práctica profesional.

Objetivo: Cuantificar el grado de cumplimento de los indicadores de seguridad en el servicio de urgencias.

Metodología: Estudio transversal sobre las urgencias con triaje realizado por el personal de enfermería en 2010 en el servicio de urgencias de un hospital comarcal. Se consideraron los siguientes indicadores: tiempo de triaje, tiempo hasta realizar un electrocardiograma (ECG) en caso de síndrome coronario agudo (SCA), tiempo hasta realizar una fibrinolisis indicada en caso de infarto agudo de miocardio (IAM), correcta identificación de los usuarios y asepsia adecuada de los catéteres endovenosos. Se realizó una descriptiva univariable mediante frecuencias.

Resultados: Se realizó en $\leq 10$ minutos el $80,3 \%$ de los 52.088 triajes y el $26 \%$ de los ECG de los 181 casos diagnosticados de SCA, y en $\leq 30$ minutos el $32 \%$ de las fibrinolisis indicadas en los 19 casos con IAM. Fue correcta la identificación del $97,6 \%$ de los pacientes y la asepsia del $90,9 \%$ de los catéteres endovenosos.

\section{ABSTRACT}

Introduction. The quality of nursing services could be defined as a convenient, personalized, humanized, continuous and efficient attention, performed by the nursing staff, according to the standards defined for professional practice.

Objective: To quantify the extent of compliance of safety indicators in the emergency department

Metotologia: Cross-sectional study in the emergency department treated a total of 55,016 emergencies in2010, excluding dropouts service without the prior triage.

Indicators are established to study, computer records at all hours of the entry of the patient, triage time nurse hours, doctor time hours of treatment, tests and time high. 
Delays are accounted for by variables such as triage time, time gate ECG (electrocardiogram) in ACS (acute coronary syndrome) and gate needle in acute myocardial infarction (AMI)Internal audit is performed and appreciated of the correct identification and asepsis of intravenous catheters.

Results: In $80.3 \%$ of cases triage nursing is made in less than 10 minutes below the quality standard of $85 \%$. Fibrinolysis is performed in AMI in less than 10 minutes ' by $32 \%$. SCA ECG is performed in less than 10 minutes to $26 \%$ of SCA visiting the emergency room, when the quality standard is $95 \%$

$97.56 \%$ of emergency were correctly identified and $90.9 \%$ of intravenous catheters maintain proper aseptic

\section{INTRODUCCIÓN}

La seguridad de los pacientes, entendida como la dimensión de la calidad asistencial que busca reducir y prevenir los riesgos asociados a la atención sanitaria, está adquiriendo un interés creciente y es uno de los ejes de la política sanitaria. Su relevancia nace del imperativo ético de primun non lacere ("antes que nada, no hacer daño"), y del convencimiento que no es aceptable que un paciente presente daños derivados de la atención sanitaria recibida, de la cual se espera que sea curadora y proporcione confort y calidad de vida. El informe To err is human ${ }^{1}$ representó un llamamiento sobre la necesidad de un sistema sanitario más seguro, ante las estimaciones que señalaban una cifra anual de hasta 98.000 muertes que podían haber sido evitadas a los Estados Unidos. En España, el Estudio ENEAS ${ }^{2}$ mostró que hasta el 9,3\% de los pacientes ingresados en un hospital sufren algún efecto adverso relacionado con la atención sanitaria. Las principales causas identificadas son el uso de medicamentos (37,4\%), la infección nosocomial $(25,4 \%)$ y la aplicación de técnicas y procedimientos $(25,0 \%)$. En un $43 \%$ de los casos, estos acontecimientos adversos podrían ser considerados como evitables.

La Organización Mundial de la Salud ha constituido la World Alliance for Patient Safety ${ }^{3}$ que lidera y promueve campañas internacionales de sensibilización en este ámbito. La primera la dedicó al lavado y higiene de manos, y ahora presenta la campaña sobre la cirugía segura. En este marco, el Ministerio de Sanidad y Consumo ha definido en el Plan de Calidad del Sistema Nacional de Salud las actuaciones prioritarias para la seguridad de los pacientes. Cataluña se ha adherido también a estas iniciativas. El Departamento de Salud ha promovido la Alianza para la Seguridad de los Pacientes ${ }^{4}$ en Cataluña como fórmula para impulsar las diferentes iniciativas y actuaciones para la mejora de la seguridad clínica. En esta iniciativa están representados y participan los agentes y los diferentes grupos de interés, como son las organizaciones de profesionales, las entidades proveedoras y las asociaciones de pacientes.

Los indicadores de calidad nos permiten objetivar situaciones relacionadas con la seguridad clínica, intentando reducir los riesgos a los que se expone el paciente que interacciona con el sistema sanitario. Un indicador es una medida cuantitativa que se utiliza como guía para controlar y valorar la calidad de aspectos importantes de la práctica asistencial. Los indicadores tienen la ventaja de medir aspectos concretos y específicos de la asistencia. Son datos válidos, fiables y objetivos, muestran resultados cuantitativos y nos dan la posibilidad de comparación al disponer de estándares de cumplimiento que nos determinan si los resultados obtenidos en nuestro servicio son correctos o no, y nos permiten realizar acciones de mejora.

El objetivo del presente estudio consistió en analizar el grado de cumplimiento de 4 de los indicadores de la Societat Catalana de Medicina d'Urgències (SCMU) y uno adaptado del VINCat ${ }^{5}$ en el servicio de urgencias del Hospital de Palamós durante 2010 que, además, 
formaron parte de los objetivos que tiene enfermería de urgencias en la dirección por objetivos (DpO).

En este ámbito, desde 1997 se aplicó la escala canadiense de triaje (Emergency Department Triage and Acuity Scale: CTAS) y desde finales del 2010 el Modelo Andorrano de Triaje $(\mathrm{MAT})^{6-8}$. Según el estándar de calidad, el personal de enfermería debe realizar en $\leq 10$ minutos el $85 \%$ de los triajes y el $95 \%$ de los electrocardiogramas (ECG) en síndrome coronario aguda (SCA), y en $\leq 30$ minutos el $100 \%$ de las fibrinolisis indicadas en caso de infarto agudo de miocardio (IAM) para reducir la mortalidad y morbilidad ${ }^{9-10}$. Además, debe ser correcta toda identificación del paciente, para evitar riesgos en la atención diagnósticoterapéutica. Ésta se realiza desde 2009 mediante pulsera identificativa en urgencias, hospitalización, zona quirúrgica y sala de partos. Finalmente, debe ser correcta toda asepsia de los catéteres endovenosos.

\section{MATERIAL Y MÉTODOS}

Estudio observacional transversal. En el servicio de urgencias se atendieron un total de 55.016 urgencias durante el 2010 con una media diaria de 150 visitas. Excluimos los abandonos del servicio sin hacer previamente el triaje.

La Societat Catalana de Medicina d'Urgències (SCMU) publicó en 2001 un trabajo en el que diseñó un total de 103 indicadores de los que seleccionó a 15 como más relevantes. Se establecen los indicadores a estudio, teniendo en cuenta estos indicadores de la SCMU y se escogen 5 indicadores que también son cuantificados por nuestro departamento de Seguridad del Paciente y que además forman parte de los objetivos que tiene enfermería de urgencias en las DpO (dirección por objetivos) del año 2010. Los indicadores y variables de nuestro estudio son: tiempo de triaje, tiempo puerta ECG en SCA, tiempo puerta aguja en IAM, identificación de paciente, asepsia de catéter endovenosa. Las fórmulas utilizadas para la cuantificación de los indicadores se pueden ver en la tabla 1.

Tabla 1. Cálculo de los indicadores evaluados.

Tiempo triaje:

Núm.paciente con tiempo de triaje $\leq 10$ minutos x 100

Núm. total de pacientes clasificados

Tiempo demora ECG en SCA:

Núm.pacientes con diagnóstico de SCA y demora de $\geq 10$ 'en la realización del ecg x 100 Núm. pacientes diagnosticados de SCA

Tiempo puerta-aguja en pacientes con IAM:

Núm. pacientes con SCA tributarios de fibrinolisis y tiempo puerta-aguja $\leq 30$ ' x 100

Núm. pacientes diagnosticados de SCA tributarios de fibrinolisis

Identicación de paciente:

Núm. pacientes identificados correctamente x 100

Número total de pacientes en urgencias

Asepsia correcta:

Núm. paciente portadores de vía periférica y correcta asepsia x 100

Número total de pacientes portadores de vía periférica 
Se registran a nivel informático todas las horas desde la entrada del paciente, hora triaje, hora enfermera box, hora médico, hora tratamientos, pruebas realizadas y hora alta.

Se contabilizan las demoras de las variables tiempo de triaje, tiempo puerta ECG (electrocardiograma) en SCA (síndrome coronario agudo) y puerta-aguja en IAM (infarto agudo de miocardio). Este registro se realiza de forma mensual por el departamento evaluación información y recerca.

Se realiza una auditoría interna 2 veces al año por parte del director de enfermería donde se valora a todos los pacientes en el censo de urgencias, la correcta identificación y se realiza otra auditoría interna 3 veces al año por parte de un miembro del comité de infecciones valorando todos los pacientes de urgencias portadores de catéter endovenoso donde se valora la correcta asepsia de los catéteres y su fijación.

El análisis estadístico se realizó con el paquete estadístico SPSS 15.0 y consistió en una descriptiva univariable mediante frecuencias

\section{RESULTADOS}

En el tiempo de triaje se valoran un total de 52088 urgencias. En el $80.3 \%$ enfermería realiza el triaje en menos de 10' no llegando al estándar de calidad que es de $85 \%$

Se valoran un total de 181 urgencias diagnosticadas de SCA. Se realiza ECG en SCA en menos de 10 ' a un $26 \%$ de los Síndromes coronarios agudos visitados en urgencias cuando el estándar de calidad es del 95\%

Se registran un total de 19 IAM candidatos a fibrinolisis. Aquí quedan excluidos todos aquellos IAM que son candidatos a angioplastia primaria o aquellos en los que la fibrinolisis no está indicada. Se realiza la fibrinolisis en los IAM en un tiempo inferior a 10' en un 32\% lejos del estándar de calidad que es un $100 \%$.

Se identifica correctamente al $97,56 \%$ de los usuarios de urgencias; el estándar de calidad es del $100 \%$.

El 90.9\% de los catéteres endovenosos mantienen una correcta asepsia.

\section{DISCUSIÓN}

El tiempo de triaje fue adecuado en el $80,3 \%$ de los casos, debiendo de ser del $85 \%$. En otros hospitales es incluso superior, como el Hospital Nostra Senyora de Meritxell $(86,9 \%)^{10}$. Puede ser causa de incumplimiento del estándar de calidad, por una parte, la toma de constantes en triaje de los grados $1,2,3$ y, según necesidad, de los grados 4 y 5 , en adultos y niños, así como en todas las pacientes que van a sala de partos, siendo suficiente la valoración de signos clínicos ${ }^{6,11,12}$. Por otra parte, sólo una enfermera realiza el triaje y, como a la vez, asume funciones en la Unidad de Soporte Vital Avanzado (USVA), su relevo produce la demora en la atención.

Respecto el tiempo hasta realizar un ECG en SCA, sólo fue adecuado en el $26 \%$, lejos del estándar de calidad del 95\% y por debajo del estudio de García-Castrillo Riesgo del estudio EVICURE ${ }^{13}{ }^{13}$. En el recuento de este indicador no se incluyen los ECG realizados en el centro de atención primaria (CAP) ni en la USVA. La mayoría de ECG los solicita la 
enfermera de triaje aunque no necesariamente los realiza. Diversos estudios desaconsejan su realización en el triaje por aumentar la demora de los triajes posteriores ${ }^{14-15}$.

El tiempo hasta realizar una fibrinolisis indicada en IAM también quedó lejos del estándar de calidad, siendo sólo correcto en el $32 \%$ de los casos. En el estudio de Epelde ${ }^{16}$ el tiempo desde la llega del enfermo al hospital hasta el inicio de la trombolisis (tiempo puerta aguja) fue de 78,7 $\pm 73,8$ minutos (rango 30-300 min). El hospital estudiado no tiene $\mathrm{UCl}$ y es el médico de urgencias quién decide su administración. Este estudio hace referencia a que así se obtienen mejores tiempos que los hospitales con UCI. Un estudio indicó como principales causas del retraso la demora al realizar e interpretar el ECG (16\% y 6\%, respectivamente), la demora de la preparación del fármaco $(8 \%)$ y la demora al realizar interconsultas ${ }^{17}$. Es importante una buena preparación de la enfermera de triaje para acelerar el proceso ${ }^{18}$.

Casi la totalidad de los pacientes fueron correctamente identificados (97,6\%). En el estudio de Martínez ${ }^{19}$ realizado en Cataluña en el 2006 se observó que de los 75 centros estudiados un $90,7 \%$ declaró utilizar algún sistema de identificación de los pacientes. Solamente el $26,7 \%$ de los centros disponían de sistemas de identificación en todos los servicios de hospitalización, pero ninguno de ellos expresa el grado de cumplimiento de esta identificación. Howanitz ${ }^{20}$ et al revisaron un total de 1.757 .730 brazaletes identificativos de 217 centros sanitarios y observaron una razón de error del 2,5\% mejorando los datos conseguidos en nuestro hospital. Las principales causas de no identificación son la no realización de triaje en la sala habilitada, que suele pasar si provienen de consultas externas, y la no reposición de la pulsera identificativa al finalizar alguna técnica, como canalización vías o gasometrías arteriales. Aunque todo el personal sanitario es responsable.

Finalmente, faltó el 9\% para cumplir con el estándar de la correcta asepsia de los catéteres endovenosos, que puede ser debido a la presión asistencial, así como a no tener un protocolo de revisión sistemática de vías periféricas en urgencias. No encontramos estudios para comparar este resultado al haber adaptado para urgencias este indicador del VinCat, aunque como podemos ver en el informe Vincat $2010^{21}$ el área de críticos es la que presenta mayor número de bacteriemias por catéteres endovenosos periféricos , un $0.10 \%$ mientras que en el área de hospitalización es de un $0.06 \%$.

Como limitaciones del estudio cabe mencionar, por un lado, la fiabilidad de las horas registradas automáticamente, pues los ordenadores podrían no estar correctamente sincronizados. Y, por otro lado, la reducida muestra obtenida de las auditorías para evaluar los indicadores sobre la correcta identificación del paciente y asepsia.

\section{CONCLUSIONES}

Dado que no se alcanzó el estándar de calidad en ninguno de los 5 indicadores analizados, deberían realizarse acciones de mejora, como aumentar la plantilla del personal de enfermería y, posteriormente, comparar los resultados obtenidos.

La utilización de estos indicadores junto con muchos otros que llevan a cabo distintos departamentos del hospital nos ofrecen un punto de mejora para la calidad y seguridad del paciente que debemos aprovechar. 


\section{REFERENCIAS BIBLIOGRÁFICAS}

${ }^{1}$ Institute of Medicine of the Nacional Academies de Washington. To Err is Human. Building a Safer Health System. Noviembre de 1999

${ }^{2}$ Ministerio de Sanidad y Consumo. Dirección General de la Agencia de Calidad del Sistema Nacional de Salud. Estudio Nacional sobre los efectos adversos ligados a la hospitalización. ENEAS, 2005. Informe de febrero de 2006

3 Alianza para la seguridad del Paciente [Citado 5 de mayo] Disponible en http://www.who.int/patientsafety/es/index

4 Avenis Donabenian.Alianza para la Seguridad del pacient en Cataluña.Definicion de linias de estrategias. [Citado 5 de mayo] Disponible en: http://www.seguretatpacient.org

${ }^{5}$ Almirante B, Bella F, Freixas N,Pujol M. Programa para reducir las bacteriemia.[Citado 5 de mayo] Disponible

en:http://www20.gencat.cat/docs/canalsalut/Minisite/VINCat/Documents/objectiu\%202/interve ncio\%20cateters/Projecte\%20prevencio\%20BRCV.doc\#_Toc251589860

${ }^{6}$ Gómez Jiménez J. Clasificación de pacientes en los servicios de urgencias y emergencias: hacia un modelo de triaje estructurado de urgencias y emergencias. Emergencias 2003;15:165-74.

${ }^{7}$ Beveridge R, Clarke B, Janes L. Canadian Emergency Department Triage and acuity scale implementation guidelines. CJEM 1999; 1 (Suppl 3): S1-S24

8 Gómez Jiménez J, Faura J, Burgues L Y Pàmies S. Gestión clínica de un servicio de urgencias hospitalario: indicadores de calidad, benchmarking y análisis de la casuística (case-mix). Gestión Hospitalaria 2004;15(1):3-12

9 The GUSTO Angiographic Investigators. The effects of tissue plasminogen activator, streptokinase, or both on coronary-artery patency, ventricular function and survival after acute myocardial infarction. N Engl J Med 1993; 329: 1615-22.

${ }^{10}$ Diercks DB, Peacock WF, Hiestand BC, Chen AY, Pollack CV jr, Kirk JD, et al. Frequency and consequences of recording an electrocardogram 10 minuts after arrival in an emergency room in non-ST-segment acute coronary syndromes. Am J Cardiol. 2006; 97:437-42

11 Roqueta Egea F. Indicadores de seguridad/riesgo en los servicios de urgencias. Monografías emergencias 2007;3:14-17

${ }^{12}$ Soler W, Gómez Muñoz M, Bragulat E, Álvarez A. El triaje: herramienta fundamental en urgencias y emergencias. An. Sist. Sanit. Navar. 2010; 33 (Supl. 1): 55-68

13 García-Castrillo L, Recuerda Martínez E, Loma-Osorio A, García-Camarero T,GarcíaCases C, Epelde Gonzalo F, Núñez Castill P, Sierra Piqueres C.Características y manejo de los pacientes con dolor torácico no traumático en los servicios de urgencias hospitalarios. Resultados del estudio EVICURE II. Emergencias 2008; 20: 391-398

${ }^{14}$ Gómez Jiménez J, Chanovas Borràs M. R., Miró O., Daza López M. , Roqueta Ejea F. , Echarte Pazos J. L., Tomás Vecina S. , Rovira Coromina J., Ciurana Roca R., Martinez Estalella G. , Nogué i Bou R. , Soto Ejarque J. M., Escalada Roig X. , Senent Llorens , A. García Alfranca F. Junta directiva i Grup de Treball de Triatge SCMU - ACMES. Triaje Estructurado en los Servicios de Urgencias. Documento de Posición de la Societat Catalana de Medicina d'Urgències i Emergències (SCMU-ACMES).2008

${ }^{15}$ XII Congrés català de urgencies i emergencies 2011

${ }^{16}$ Epelde Gonzalo F y Grupo de Trabajo de Cardiopatía Isquémica. Sociedad Española de Medicina de Urgencias(SEMES). Situación del tratamiento trombolítico del infarto agudo de miocardio en los Servicios de Urgencias de Cataluña. An Med Interna (Madrid) 2003; 20: 7577.

${ }^{17}$ Busto Colina Y, Escolana Ramos A, Busto Santiesteban G, Busto Colina Y. Causas de demora en los tiempos de espera para el tratamiento trombolítico sistémico con estreptoquinasa recombinante en el hospital de amancio. Años 1998-2004.Rev Cubana Med Disponible en : http://www.sld.cu/galerias/pdf/sitios/urgencia/080. 
${ }^{18}$ Loro Sancho N, Sancho Sánchez Maㅡ, Sancho Sánchez Maㅜ, Sierra Talamantes C. Atención de enfermería en el IAM en la Unidad de Urgencias. Rev. Enferm.Cardio.2001.25:41-4.

19 Martínez L, Gimeno V, Anglès R, Bañeres J, Torralba L y Manzanera R. Sistemas de identificación de pacientes en hospitales de Catalunya. Rev Calidad Asistencial. 2008;23(4):158-63

${ }^{20}$ Howanitz PJ, Renner SW, Walsh MK. Continuous wristband monitoring over 2 years decreases identification errors. Arch Pathol Lab Med. 2002;126:809-15.

${ }^{21}$ Programa de Vigilància de les Infeccions Nosocomials als Hospitals de Catalunya. Vincat informe 2010.[Citado 6 junio]. Disponible en:

http://www20.gencat.cat/docs/canalsalut/Minisite/VINCat/Documents/Informes/Informe\%2020 10.pdf 